\title{
BAJA FRECUENCIA DE PRESCRIPCIÓN DE PROFILAXIS PARA OSTEOPOROSIS EN PACIENTES EN TRATAMIENTO CRÓNICO CON CORTICOIDES EN COLOMBIA
}

\author{
Jorge Machado-Alba1,2,a, Verónica Alzate-Carvaja|3 ${ }^{3,4, b}$, Álvaro Mondragón-Cardona ${ }^{3,4, b}$, \\ Carlos Eduardo Jiménez-Canizales 3 ,4,b
}

RESUMEN

Objetivos. Identificar la frecuencia de uso de medicamentos para prevención de osteoporosis inducida por corticoides (OIC) en pacientes que reciben corticoides por un periodo mayor a tres meses y que son afiliados al Sistema General de Seguridad Social en Salud de Colombia. Materiales y métodos. Estudio de tipo transversal. Se utilizó información sobre, las personas afiliadas al Sistema General de Seguridad Social en Salud de Colombia (3,7 millones). Se incluyeron en el trabajo los usuarios de todas las edades y sexos a los cuales se les indicó algún glucocorticoide entre el 1 de agosto y 30 de noviembre de 2011.Se identificaron variables sociodemográficas y las características de prescripción de glucocorticoides y medicamentos para profilaxis de OIC con dosis expresadas en dosis diarias definidas (DDD). Resultados. Se obtuvo una base de datos de 255568 prescripciones de glucocorticoides, de los cuales 1837 pacientes recibían algún glucocorticoide de manera crónica. Predominaron pacientes de sexo femenino $(60,2 \%)$, edad promedio de $55,2 \pm 16,9$ años distribuidos en 65 ciudades del país. El glucocorticoide más utilizado fue prednisolona en 1546 (84,1\%), mientras que el medicamento para profilaxis más prescrito fue calcitriol $(67,1 \%)$. Se encontraron 994 casos $(54,2 \%)$ que no estaban recibiendo profilaxis para osteoporosis a pesar de requerirlo. Conclusiones. Existe un bajo empleo de profilaxis para OIC. Se recomienda implementar acciones de farmacovigilancia que permitan identificar problemas relacionados con medicamentos para prevenir eventos adversos y optimizar recursos, anticipándose a la aparición de complicaciones para el paciente.

Palabrasclave: Glucocorticoides; Calcitriol;Alendronato; Osteoporosis; Prevención secundaria; Farmacoepidemiología; Farmacovigilancia (fuente: DeCS BIREME).

\section{LOW FREQUENCY OF PROPHYLAXIS PRESCRIPTION FOR OSTEOPOROSIS IN PATIENTS RECEIVING CHRONIC TREATMENT WITH CORTICOSTEROIDS IN COLOMBIA}

\begin{abstract}
Objective. To identify the frequency of drug use for the prevention of corticosteroid induced osteoporosis (CIO) among patients using corticosteroids for more than three months affiliated to the General Social Health Security System of Colombia. Materials and methods. Cross-sectional study. Information about people affiliated to the General Social Health Security System of Colombia (3.7 million) was used. This study included men and women of all ages having been prescribed any glucocorticoid from August 1 to November 30, 2011. Sociodemographic variables and the characteristics of of the glucocorticoid prescription and $\mathrm{ClO}$ prophylaxis drugs in a defined daily dose (DDD) were identified. Results. A database of 255,568 prescriptions of glucocorticoids was obtained, with a total of 1,837 patients receiving some glucocorticoid chronically. The majority of participants were females $(60.2 \%)$, with an average age of $55.2 \pm 16.9$ years distributed in 65 cities of the country. Prednisolone was the most commonly used glucocorticoid (1,546 patients, 84.1\%), whereas the most prescribed prophylaxis drug used was calcitriol (67.1\%). Despite the need to receive prophylaxis, 994 cases $(54.2 \%)$ were not receiving it. Conclusions. There is a poor utilization of $\mathrm{ClO}$ prophylaxis. The implementation of drug surveillance actions that allow the identification of problems related to these drugs in order to prevent adverse events and optimize resources is recommended, thus anticipating the emergence of complications in the patients.
\end{abstract}

Key words: Glucocorticoids; Calcitriol; Alendronate; Osteoporosis; Secondary prevention; Pharmacoepidemiology; Pharmacovigilance (source: MeSH NLM).

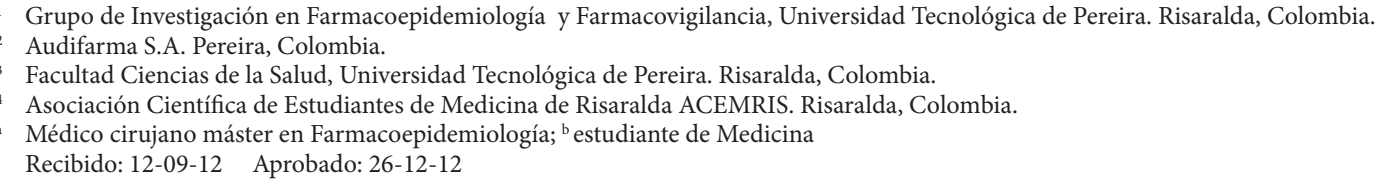

Citar como: Machado-Alba J, Alzate-Carvajal V, Mondragón-Cardona A, Jiménez-Canizales CE. Baja frecuencia de prescripción de profilaxis para osteoporosis en pacientes en tratamiento crónico con corticoides en Colombia. Rev Peru Med Exp Salud Publica. 2013;30(1):26-30. 


\section{INTRODUCCIÓN}

Según la Organización Mundial de la Salud (OMS), la osteoporosis se define en base a la medición de la densidad ósea en la columna lumbar o el cuello del fémur (valor de índice $T$ inferior a 2,5 desviaciones estándar) ${ }^{(1)}$. Se estima que la prevalencia de osteoporosis en personas mayores de 50 años es del $14 \%{ }^{(2)}$.

La ingesta crónica de corticoides puede producir osteoporosis, lo que lleva a aumentar el riesgo de fracturas (cadera, fémur y columna vertebral); mayor número de hospitalizaciones; discapacidad, y pérdida de tiempo laboral ${ }^{(1)}$. Se estima que más del $50 \%$ de pacientes que reciben tratamiento con corticoides presentan riesgo de osteoporosis y menos del $25 \%$ de estos pacientes reciben algún tipo de prevención farmacológica ${ }^{(3)}$.

Se recomienda administrar profilaxis para osteoporosis inducida por corticoides (OIC) en los siguientes casos: i) paciente que recibe dosis superiores a $2,5 \mathrm{mg} / \mathrm{día}$ de prednisona o su equivalente (si son mujeres posmenopáusicas) por más de tres meses; ii) mujeres premenopáusicas o varones que reciben dosis de $7,5 \mathrm{mg} /$ día, y iii) mujeres premenopáusicas o varones con una puntuación $\mathrm{T}$ inferior a $-1,5$ que reciben dosis de $5 \mathrm{mg} / \mathrm{dí}^{(4)}$.

Para el tratamiento y la prevención de la OIC se recomienda controlar el peso, realizar ejercicio habitualmente y abolir el consumo de tabaco y alcohol ${ }^{(5)}$. El manejo farmacológico con bifosfonatos ha evidenciado que aumenta la densidad mineral ósea en un 3,8 a $5,4 \%$, y previene la pérdida de densidad ósea en hasta $3,7 \%{ }^{(6)}$. Por otro lado, medicamentos como la vitamina $\mathrm{D}$, la calcitonina $\mathrm{y}$ las terapias de remplazo hormonal incrementan la densidad ósea desde 1,9 a 3,8\%, reduciendo así los riegos de fractura e incapacidad hasta en $70 \%{ }^{(7)}$.

Existen diferentes estrategias de farmacovigilancia, todas ellas orientadas a favorecer el mejor uso de los medicamentos al interior de las instituciones asistenciales, dado que al detectar el riesgo de reacciones adversas permite evaluar las prácticas de prescripción, dispensación, administración y consumo de medicamentos ${ }^{(8,9)}$. En la literatura científica revisada no se encontraron estudios similares hechos en Colombia, y debido al alto porcentaje de pacientes que utilizan corticoides y que pueden no recibir profilaxis para prevenir la osteoporosis ${ }^{(3)}$, el objetivo del estudio fue identificar la frecuencia en el uso de medicamentos para la prevención de la OIC en pacientes que los reciben por más de tres meses pertenecientes al Sistema General de Seguridad Social en Salud de Colombia (SGSSS).

\section{MATERIALES Y MÉTODOS}

Se realizó un estudio transversal sobre la prescripción de corticoides por un periodo mayor a tres meses en una población de 3,7 millones de personas $(20,4 \%$ de Ios afiliados al SGSSS y $8,2 \%$ de la población colombiana afiliada a empresas promotoras de salud e instituciones prestadoras de servicios de salud) ${ }^{(10)}$. La población procedía de 89 municipios que cuentan entre 20000 y 7,5 millones de habitantes. El $80 \%$ de los pacientes provenía de Barranquilla, Bogotá, Bucaramanga, Cali, Cartagena, Ibagué, Manizales, Medellín y Pereira. Se incluyeron los pacientes de todas las edades y de ambos sexos, a quienes se les dispensó algún corticoide por más de tres meses en el periodo comprendido entre el 1 de agosto y el 30 de noviembre de 2011.

A partir de la información sobre consumo de medicamentos registrada por la empresa encargada de dispensar los medicamentos a la población afiliada, se confeccionó una base de datos PostgreSQL y se utilizó el aplicativo Delphi para Windows ${ }^{\circledR}$. Se emplea la herramienta Business Object sobre una plataforma en Oracle donde se almacenan todas las dispensaciones que se hacen a diario a los pacientes afiliados al SGSSS, en este caso a lo largo del periodo de estudio, y que visualiza las fórmulas que previamente han sido puestas en un formato digital. Todos los registros son revisados por profesionales especializados del departamento de farmacoepidemiología, que involucran los momentos de la dispensación, revisión y facturación.

Se registraron las siguientes variables: edad, sexo, corticoides utilizados por más de tres meses (prednisona, prednisolona, metilprednisolona, triamcinolona, betametasona, dexametasona, hidrocortisona, etc.) y medicamentos utilizados para prevenir osteoporosis (bifosfonatos, paratohormona, calcitonina, vitamina D calcio, carbonato de calcio, estrógenos, progestágenos). Para la cuantificación de uso se ha utilizado como unidad técnica la dosis diaria definida (DDD) recomendada por la OMS como estándar internacional para la realización de estudios farmacoepidemiológicos. En este caso se empleó el indicador promedio de la DDD de cada medicamento que estaban recibiendo los pacientes al mes, y el número de DDD dispensadas (nDDD) al mes por cada 100000 afiliados.

Se consideraron todos los pacientes que recibían o no medicamentos profilácticos para OIC. Se realizó un análisis estadístico descriptivo. De los pacientes identificados por el riesgo de OIC se notificó al responsable de la atención sanitaria para la evaluación y toma de decisiones.

El protocolo fue sometido a revisión científica y bioética de la Facultad de Ciencias de la Salud de la Universidad 
Tecnológica de Pereira en la categoría de investigación sin riesgo, según la resolución 008430 de 1993 del Ministerio de Salud de Colombia. Además, se realizó la notificación del riesgo de cada paciente a los directores científicos mediante un documento que respaldaba el análisis y en el que se hicieron recomendaciones para valorar la necesidad de la continuidad de la corticoterapia o de la inclusión de un fármaco que previniese el posible desarrollo de osteoporosis.

\section{RESULTADOS}

Se obtuvo una base de datos con 255568 prescripciones en el periodo total de cuatro meses, donde se encontró que $1837(0,7 \%)$ pacientes recibieron algún corticoide de manera crónica, de los cuales el 60,2\% fueron mujeres con edad promedio de 55,2 \pm 16,9 años. Se encontraron pacientes de 36 empresas promotoras de salud e instituciones prestadoras de servicios de salud (EPS o IPS) distribuidos en 65 ciudades del país. La ciudad con mayor número de pacientes que recibió corticoides fue Bogotá (903 pacientes), seguido de Manizales (138), Cali (126), Pereira (115), Barranquilla (82) y Medellín (74).

En estos pacientes, se registraron 1917 prescripciones de corticoides. Se encontró prednisolona en 1571 prescripciones, dexametasona en 157, deflazacort en 134, metilprednisolona en 28 , betametasona en 18 , hidrocortisona en 7 y triamcinolona en 2. Algunos pacientes recibieron combinaciones de corticoides: dexametasona + betametasona + prednisolona (4), dexametasona + prednisolona (2), dexametasona + betametasona (1), dexametasona + betametasona + prednisolona + metilprednisolona (1) y dexametasona + triamcinolona + prednisolona + betametasona (1).

En relación a la prescripción de algún medicamento como profilaxis de osteoporosis, de los 1837 pacientes que recibieron corticoides de forma crónica, se encontró 1050 prescripciones provenientes de 843 pacientes $(45,8 \%)$ que los recibían en forma continua.

El medicamento más empleado fue el calcitriol (705 prescripciones), seguido de alendronato (197), ácido ibandrónico (48), risedronato (43), terapia de remplazo hormonal (39), calcio (13), paricalcitol (3) y calcitonina (2). Los indicadores de consumo de los corticoides y de los medicamentos utilizados para la profilaxis de osteoporosis se presentan en la Tabla 1 en forma de nDDD durante los meses de consumo y, además, en forma de dosis diarias definidas por 100000 habitantes. Sobre la ciudad de procedencia del grupo de 994 (54,2\%) pacientes que no recibieron profilaxis para osteoporosis, la mayoría provenía de Bogotá $(38,1 \%)$, Manizales $(11,0 \%)$ y Cali $(8,7 \%)$.
Tabla 1. Número de dosis diarias definidas de glucocorticoides y medicamentos para la profilaxis de osteoporosis en una población afiliada al Sistema General de Seguridad Social en Salud de Colombia, 2011.

\begin{tabular}{|c|c|c|}
\hline Medicamento & $\mathrm{nDDD}$ & $\begin{array}{c}\text { DDD X } \\
100000 \\
\text { habitantes }\end{array}$ \\
\hline Alendronato tableta $70 \mathrm{mg}$ & 2,1 & 272,3 \\
\hline Prednisolona tableta $5 \mathrm{mg}$ & 0,6 & 254,4 \\
\hline Dexametasona solución iny 8 mg & 0,4 & 211,1 \\
\hline $\begin{array}{l}\text { Medroxiprogesterona } \\
\text { suspensión iny } 50 \mathrm{mg}\end{array}$ & 1,3 & 183,1 \\
\hline Prednisolona tableta $50 \mathrm{mg}$ & 1,6 & 153,4 \\
\hline Calcitriol capsula $0,25 \mathrm{mcg}$ & 0,3 & 80,8 \\
\hline Dexametasona solución iny 4 mg & 0,2 & 69,2 \\
\hline Calcitriol capsula $0,5 \mathrm{mcg}$ & 0,6 & 63,4 \\
\hline Risedronato tableta 35 mg & 5,9 & 55,7 \\
\hline $\begin{array}{l}\text { Metilprednisolona polvo reconstituir } \\
500 \mathrm{mg}\end{array}$ & 2,1 & 52,0 \\
\hline $\begin{array}{l}\text { Estrógenos conjugados tableta } \\
0,625 \mathrm{mg}\end{array}$ & 1,1 & 51,5 \\
\hline $\begin{array}{l}\text { Betametasonafosfato/betametasona- } \\
\text { acetato }\end{array}$ & 0,2 & 48,7 \\
\hline Medroxiprogesterona tableta 5 mg & 0,8 & 44,2 \\
\hline Betametasona solución iny 4 mg & 0,2 & 33,3 \\
\hline Ácido ibandronico tableta 150 mg & 1,1 & 30,9 \\
\hline Risedronato sódico tableta 150 mg & 1,1 & 11,9 \\
\hline $\begin{array}{l}\text { Alendronato + colecalciferol } \\
\text { (vitamina D3) }\end{array}$ & 1,1 & 1,4 \\
\hline
\end{tabular}

DDD: dosis diarias definidas; iny: inyectable

nDDD: Número de DDD que en promedio recibe cada paciente

\section{DISCUSIÓN}

Los importantes efectos antinflamatorios e inmunosupresores de los corticoides los ubica en el grupo de medicamentos más usados en la práctica clínica. Se estima que 1 al $3 \%$ de los adultos usa algún corticoide de los cuales $22,1 \%$ lo hace por más de seis meses ${ }^{(11)}$, datos similares a los del presente estudio en el cual se evidenció un importante número de prescripciones en cuatro meses (255 568 pacientes que corresponden al 1\% de la población susceptible) y un uso prolongado en 1837 pacientes.

El incremento del riesgo de fracturas vertebrales y no vertebrales se asocia con dosis de prednisolona o sus equivalentes entre 2,5 y 7,5 mg/día, y en este trabajo se encontraron prescripciones con dosis superiores en pacientes sin profilaxis para osteoporosis, lo que podría aumentar el riesgo de fracturas ${ }^{(12,13)}$. Se ha estimado que más de un $30 \%$ de los pacientes prescritos con glucocorticoides desarrollarán fracturas por fragilidad ósea, siendo destacable que el riesgo de fracturas vertebrales es particularmente elevado y se ha estimado entre 2 a 5 veces mayor dependiendo la dosis diaria recibida ${ }^{(4)}$. Sin embargo, debido a que un importante número de pacientes están recibiendo DDD de prednisolona menores a las 
recomendadas debe considerarse la posibilidad que esté siendo subdosificada, o que estos pacientes respondan a dosis inferiores a las utilizadas internacionalmente o incluso que tengan un menor riesgo de OIC al estimado.

En un estudio realizado en el Caribe colombiano se encontró que de la totalidad de los pacientes estudiados con prescripción de corticoides la mayoría pertenecían al sexo femenino, similar a lo encontrado en nuestro trabajo, datos que pueden indicar que un importante porcentaje de la población estudiada presenta como factor de riesgo las alteraciones hormonales dadas por la posmenopausia ${ }^{(14-16)}$, entre otros factores de riesgo.

Se encontró, además, que $54 \%$ de los casos no recibían algún medicamento para la prevención de la OIC, dato menor comparado con un estudio realizado en Inglaterra el cual halló que $64,7 \%$ de los pacientes que reciben corticoides de manera crónica, no se les administraba una correcta profilaxis de acuerdo a las guías internacionales ${ }^{(17)}$. Un estudio realizado en Colombia en 2005 señaló que menos del $10 \%$ de los pacientes con corticoides de uso crónico recibían terapia profiláctica, lo que sitúa el porcentaje acá encontrado en un punto intermedio donde se evidencia un aumento del uso de alguna medida farmacológica para la profilaxis de OIC, pero que no deja de ser preocupante ${ }^{(14)}$

Dentro de las medidas farmacológicas se ha recomendado iniciar la ingesta de calcio de 1000 a 1500 mg por día y vitamina D de 800 a 1000 UI por día ${ }^{(18,19)}$. Un estudio encontró que el $17 \%$ de los pacientes que solo recibían calcio más vitamina $\mathrm{D}$ como profilaxis para OIC, presentaron fracturas vertebrales en el primer año de tratamiento ${ }^{(13)}$. El uso de bifosfonatos como alendronato o risedronato son recomendados según la estratificación de riesgo basada en la herramienta para el abordaje del riesgo de fractura FRAX diseñada por la Organización Mundial de la Salud (OMS) ${ }^{(12,18)}$; y el alendronato ha sido identificado como la medida farmacológica con mayor efectividad en la prevención de la OIC y en la recuperación de la disminución de la densidad mineral ósea, el cual puede ser empleado con o sin estratificación de riesgo de fractura, dado que los potenciales beneficios superan los riesgos de su exposición ${ }^{(4)}$. El presente estudio señala que el calcitriol como combinación de calcio y vitamina $D$ es la medida farmacológica más empleada $(67,1 \%)$, seguido por el alendronato, apenas utilizado en el 18,8\% de los casos, por lo cual este último estaría subempleando, dada la evidencia científica que señala su superioridad en la prevención y manejo de este riesgo ${ }^{(12)}$.

El hallazgo de estos casos justifica las actividades de fármacovigilancia, en especial para reducir la probabilidad de eventos adversos en aquellos pacientes que tienen factores de riesgo para OIC y fracturas ${ }^{(8,12)}$. Un paso subsiguiente a la identificación de los casos por medio de la fármacovigilancia, consiste en educar al personal sanitario en la importancia de emplear estrategias preventivas para la OIC; para que establezcan medidas no farmacológicas como identificar y modificar los diferentes factores de riesgo en los pacientes, aconsejando disminuir el hábito de fumar; promoviendo la práctica de actividad física diaria y disminuyendo el consumo de alcohol, y mediante medidas farmacológicas como manejar dosis mínimas efectivas por periodos lo más cortos posibles, suministrar terapia hormonal en caso de necesitarlo, suministrar suplementos vitamínicos y emplear medicamentos específicos como los bifosfonatos, y garantizar la adherencia $(4,6,12,20)$.

La propuesta de intervención consiste básicamente en informar del riesgo a cada asegurador y médico prescriptor, para que cada paciente sea valorado por ellos y se tomen las decisiones frente al uso de medidas y medicamentos que puedan prevenir el desarrollo de osteoporosis. Debe contemplarse el empleo de profilaxis cuando se va a administrar una dosis mayor de prednisolona al día o su equivalente farmacológico por más de tres meses, o en aquellos pacientes identificados según grupo de riesgo. Además, debe considerarse cuando haya otros factores de riesgo como la edad (mujeres posmenopáusicas, hombres mayores de 50 años); hábitos (alimentación deficiente, tabaquismo y alcohol); antecedentes de fracturas por fragilidad, y enfermedades concomitantes ${ }^{(5,12,21,22)}$.

Este trabajo presenta algunas limitaciones, las cuales pueden superarse con otros estudios de utilización de medicamentos, correspondientes a la segunda fase de esta línea de investigación. Teniendo en cuenta que por la metodología del estudio se recolecta la información de una base de datos de prescripciones, es necesario realizar investigaciones posteriores en los cuales se logren determinar el estado de la densidad mineral ósea en los pacientes con y sin profilaxis para osteoporosis, los intervalos de uso de los medicamentos para la profilaxis de la osteoporosis, la incidencia de OIC y la adherencia a la profilaxis de la OIC. Dado que se desconocía el estado de la densidad mineral ósea de los pacientes, de las condiciones asociadas y los factores de riesgo, no se puede afirmar si la prescripción era la adecuada. Por otro lado, teniendo en cuenta que se trata de una población cautiva que recibe medicamentos de un listado específico, los resultados y conclusiones son aplicables solo para poblaciones similares.

Como recomendaciones, los prestadores logísticos de la distribución deben involucrarse en toda la cadena del medicamento, para velar por el buen uso del recurso farmacéutico, lo cual podría reducir el riesgo de aparición de problemas negativos asociados con la medicación. Además, se debe evaluar el riesgo de fracturas y otros 
factores de riesgo asociados en los pacientes con uso crónico de corticoides, así como la adherencia farmacológica a la medicación profiláctica y, finalmente, lo más importante es la educación de los pacientes (ello incluye cambios en los estilos de vida y en la dieta), y de los prescriptores, como parte de programas estructurados de prevención y seguridad del uso de medicamentos ${ }^{(10,18,23)}$.

En conclusión, la profilaxis para la OIC está siendo insuficientemente utilizada en los pacientes que tienen riesgo de desarrollarla, además, se está valorando poco dicho riesgo, y cuando se emplean medicamentos profilácticos se seleccionan aquellos que no han demostrado la mejor evidencia de protección, por lo que se deberían usar más bifosfonatos que derivados del calcio, siguiendo las recomendaciones internacionales.

Contribuciones de autoría: VAC, AMC, CJC han participado en la concepción del artículo, redacción del proyecto, análisis de resultados, redacción de estos y de la discusión. JEMA ha participado en la redacción del proyecto, recolección de la información, análisis de información, descripción de resultados, discusión, revisión crítica del artículo, y evaluación de la versión final del manuscrito. Todos los autores aprueban la versión final del artículo.

Fuentes de financiamiento: Universidad Tecnológica de Pereira y Audifarma S.A.

Conflictos de interés: los autores declaran no tener conflictos de interés en la publicación de este artículo.

\section{REFERENCIAS BIBLIOGRÁFICAS}

1. Lidofsky S, Smith J. Glucocorticoid-induced osteoporosis in inflammatory bowel disease. Med Health R I. 2009;92(4):128-30.

2. Hadji P, Klein S, Gothe H, Haussler B, Kless T, Schmidt T, et al. The Epidemiology of Osteoporosis-Bone Evaluation Study (BEST): An Analysis of Routine Health Insurance Data. Dtsch Arztebl Int. 2013;110(4):52-7.

3. Larroudé MS, Man Z. Osteoporosis inducida por corticoides. Rev Colomb Reumatol. 2004;11(2):111-39.

4. den Uyl D, Bultink IE, Lems WF. Advances in glucocorticoid-induced osteoporosis. Curr Rheumatol Rep. 2011;13(3):233-40.

5. Kirigaya D, Nakayama T, Ishizaki T, Ikeda S, Satoh T. Management and treatment of osteoporosis in patients receiving long-term glucocorticoid treatment: current status of adherence to clinical guidelines and related factors. Intern Med. 2011;50(22):2793-800.

6. Iwamoto J, Takeda T, Sato Y. Prevention and treatment of corticosteroidinduced osteoporosis. Yonsei Med J. 2005;46(4):456-63.

7. Woolf AD. An update on glucocorticoidinduced osteoporosis. Curr Opin Rheumatol. 2007;19(4):370-5.

8. Faus MJ AP, Martinez-Martinez F. Atención farmacéutica: conceptos, proceso y casos prácticos. Madrid: Ergon. 2008. p. 139-62.

9. Calderón Ospina CA, Urbina Bonilla AP. La Farmacovigilancia en los últimos 10 años: actualización de conceptos y clasificaciones. Logros y retos para el futuro en Colombia. Méd UIS. 2011;24(1):57-73.
10. McDonough RP, Doucette WR, Kumbera P, Klepser DG. An evaluation of managing and educating patients on the risk of glucocorticoid-induced osteoporosis. Value Health. 2005;8(1):24-31.

11. Deal CL. Recent recommendations on steroid-induced osteoporosis: More targeted, but more complicated. Cleve Clin J Med. 2013; 80(2):117-25.

12. Grossman JM, Gordon R, Ranganath VK, Deal C, Caplan L, Chen W, et al. American College of Rheumatology 2010 recommendations for the prevention and treatment of glucocorticoid-induced osteoporosis. Arthritis Care Res (Hoboken). 2010;62(11):1515-26.

13. Boling EP. Secondary osteoporosis: underlying disease and the risk for glucocorticoid-induced osteoporosis. Clin Ther. 2004;26(1):1-14.

14. Caballero-Uribe CV, Acosta JG, Andrade FM, Hernández EA, Peñuela M. Prevención, diagnóstico y tratamiento de la osteoporosis inducida por corticoides (OIC). Patrones de conducta en reumatólogos del caribe colombiano. Rev. Colomb. Reumatol. 2005;12(2):159-64.

15. Bączyk G, Opala T, Kleka P, Chuchracki M. Multifactorial analysis of risk factors for reduced bone mineral density among postmenopausal women. Arch Med Sci. 2012;8(2):332-41.

16. Hart SR, Green B. Osteoporosis prophylaxis during corticosteroid treatment: failure to prescribe. Postgrad Med J. 2002;78(918):242-3.

17. Dawson-Hughes B, Tosteson AN, Melton LJ 3rd, Baim S, Favus MJ, Khosla S, et al. Implications of absolute fracture risk assessment for osteoporosis practice guidelines in the USA. Osteoporos Int. 2008;19(4):449-58.

18. den Uyl D, Bultink IE, Lems WF. Glucocorticoid-induced osteoporosis. Clin Exp Rheumatol. 2011;29(5 Suppl 68):S93-8.

19. Bachmeyer C, Gauthier M. How to prevent glucocorticoid-induced osteoporosis. Cleve Clin J Med. 2010;77(11):762

20. Netelenbos JC, Geusens PP, Ypma G, Buijs SJ. Adherence and profile of nonpersistence in patients treated for osteoporosis--a large-scale, long-term retrospective study in The Netherlands. Osteoporosis Int. 2011;22(5):1537-46.

21. Homik J, Suarez-Almazor ME, Shea B, Cranney A, Wells G, Tugwell P. Calcium and vitamin $\mathrm{D}$ for corticosteroid-induced osteoporosis. Cochrane Database Syst Rev. 2000(2):CD000952.

22. Sosa Henríquez M, Díaz Curiel M, Díez Pérez A, Gómez Alonso C, González Macías J, Farrerons Minguella J, et al. Guide for the prevention and treatment of glucocorticoid-induced osteoporosis of the Spanish Society of Internal Medicine. Rev Clin Esp. 2008;208(1):33-45.

23. de Bekker-Grob EW, Essink-Bot ML, Meerding WJ, Koes BW, Steyerberg EW. Preferences of GPs and patients for preventive osteoporosis drug treatment: a discrete-choice experiment. Pharmacoeconomics. 2009;27(3):211-9.

Correspondencia: Jorge Enrique Machado-Alba Dirección: Calle 105 No. 14-140, Pereira,

Risaralda, Colombia.

Teléfono: (57) 3108326970

Correoelectrónico:machado@utp.edu.co 